\title{
FUENTES PARA LA ELABORACIÓN DE UN MAPA EDITORIAL DE LIBROS DE TEXTO EN ESPAÑ ${ }^{1}$
}

\author{
Miguel Beas Miranda² \\ Erika González García ${ }^{3}$
}

\section{RESUMEN}

Independientemente del debate sobre el uso o abuso de los libros de texto y el de su utilidad como recursos educativos, su repercusión en los centros escolares está directamente relacionada con la cuota de mercado de las editoriales. En España no se publican los datos de la difusión que tiene cada libro de texto, por eso el objetivo de este artículo es analizar cada una de las fuentes de las que podemos extraer alguna información relacionada con este tema. Examinaremos las fuentes provenientes tanto de los organismos y entidades públicas, como del propio sector editorial, en un ámbito en el que se demanda más información y transparencia. Entre las conclusiones, las fuentes analizadas aportan datos globales de las editoriales, pero no de cada editorial, ni la

\footnotetext{
${ }^{1}$ Este estudio tuvo su origen en un proyecto de investigación I+D+i Ciudadanía, identidades complejas y cultura política en los manuales escolares españoles (1978-2006) dirigido por Gabriela Ossenbach Sauter (SEJ-66097/EDU) y otro homónimo de ámbito andaluz cuyo investigador principal fue Miguel Beas Miranda (Po7-SEJ-03305).

2 Universidad de Granada (UGR), Granada, Espanha.

3 Universidad de Granada (UGR), Granada, Espanha.
} 
concreción por materias, ni por niveles educativos; el mercado lo dominan las llamadas empresas muy grandes y grandes. Este estudio puede ser muy útil para quienes investiguen sobre cualquier campo relacionado con los libros de texto y tengan en cuenta sus cifras de comercialización.

Palabras clave: educación, libros de texto, recursos educativos, editoriales, mapa editorial.

\title{
FONTES PARA O DESENVOLVIMENTO DE UM MAPA EDITORIAL DE LIVROS DIDÁTICOS EM ESPANHA
}

\section{RESUMO}

Independentemente da discussão à volta do uso ou abuso de livros didácticos, e da sua utilidade como recursos educacionais, a repercussão que estes têm nas escolas está directamente relacionada com a quota de mercado de cada uma das editoras. Na Espanha, não se publicam os dados sobre a distribuição de cada livro didático; por isso, o objetivo deste estudo é analisar cada uma das fontes das quais possamos extrair alguma informação relacionada com essa questão. Serão examinadas fontes quer de organismos e entidades públicas, quer do sector editorial, numa área em é exigida mais informação e transparência. Entre as conclusões verifica-se que as fontes de dados analisadas fornecem dados globais das editoras, mas não de cada editora em particular, assim como não detalham dados por assunto nem por nível de ensino; o mercado é dominado por empresas grandes e muito grandes. Este estudo pode revelar-se muito útil para todos aqueles que investigam em qualquer campo relacionado com livros didácticos e que pretendam ter em conta dados relacionados com a sua comercialização.

Palavras-chave: educação, livros didáticos, recursos educacionais, editoras, mapa editorial.

\section{SOURCES FOR THE DEVELOPMENT OF A PUBLISHING MAP OF TEXTBOOKS IN SPAIN}

\begin{abstract}
Regardless of the debate over the use or abuse of textbooks and their usefulness as educational resources, their impact on schools is directly related to the market shares of publishers. In Spain, details of the distribution of each text book are not given. Therefore, the aim of this article is to analyze each of the sources from which we can extrapolate some information about this subject. We examine the sources from both agencies and public entities, such as the publishing industry itself, in an area in which more information and transparency is demanded. Among the findings, the analyzed sources provide global data of the publishers, but not of each publisher, nor the concretion by subject nor by educational levels; the declared large and very large companies dominate the market. This study is very useful for those who investigate any field related to textbooks and take into account their figures of commercialization.
\end{abstract}

Keywords: education, textbooks, educational resources, publisher, publishing map. 


\section{SOURCES POUR L'ÉLABORATION D'UNE CARTOGRAPHIE DES MAISONS D'ÉDITION DES MANUELS SCOLAIRES EN ESPAGNE}

\section{RÉSUMÉ}

Sans préjudice du débat sur l'usage ou l'abus les livres scolaires et de leur efficacité comme outil pédagogique, leur répercussion dans les centres scolaires est directement liée à la part de marché des maisons d'édition. En Espagne on ne publie pas les données diffusion de chaque manuel, raison por laquelle, l'objectif de cet article essaye d'analyser chacune des sources dont il est possible d' extraire une information relative à ce sujet. Nous examinerons les sources issues aussi bien des organismes et des collectivités publiques, comme du propre secteur de l'édition, dans un domaine où on exige plus d'information et transparence. Entre les conclusions, les sources analysées, apportent des données globales des maisons d'édition, mais pas de chacune d'entre elles, ni de précisions sur les matières, ni des niveaux éducatifs; ce sont les grandes, ou très grandes entreprises, celles qui dominent le marché. Cette étude peut être très utile pour les personnes qui font des recherches sur n'importe quel aspect concernant les livres scolaires et qui doivent prendre compte des chiffres de la commercialisation.

Mots-clés: éducation, livres scolaires, ressources pédagogiques, maisons d'édition, cartographie des maisons d'édition. 


\section{INTRODUCCIÓN}

La incidencia de los libros de texto en el ámbito educativo no es fácil de desvelar: porque el uso y la dependencia metodológica que se puede tener de ellos es muy diversa, por el contrapeso cultural que ejercen los medios de comunicación y los contextos sociales y porque frecuentemente no se realiza un mapa editorial que nos muestre qué población escolar utiliza cada libro de texto. Se pueden efectuar estudios epistemológicos, análisis de elementos curriculares, investigaciones axiológicas o dilucidar sobre representaciones cognitivas como si todos los libros de texto de cualquier editorial tuviesen la misma repercusión. Parece poco discutible que si estas investigaciones no tienen en cuenta la difusión de los libros analizados, el alcance de las conclusiones que se obtengan carece de validez.

Con frecuencia también, nos encontrarnos con trabajos que intentan realizar el mapa editorial basándose en fuentes erróneas para este fin; y lo son, porque no informan sobre las conclusiones que los distintos autores extraen. Así, por ejemplo, que una determinada editorial, conocida porque la mayor parte de sus libros son de texto, inscriba un número elevado de ISBN no implica que todos sus libros sean manuales escolares ni que sean los más difundidos en los centros escolares. En este sentido, nuestro objetivo es analizar las fuentes relacionadas con el sector editorial de libros de texto sopesando su valía para la elaboración de un mapa editorial.

Para hacer cualquier investigación que tenga como objeto el análisis de los libros de texto, está claro que no se pueden analizar los miles de títulos de Infantil, Primaria y Secundaria que se editan cada año y menos aún si lo que se pretende es hacer un estudio que comprenda un período de tiempo de varios años. A modo de ejemplo y grosso modo, teniendo en cuenta tan solo los libros correspondientes a cuatro materias por curso, por ocho niveles educativos, por una media de quince editoriales y por treinta años, la suma total de títulos es de 14.40o. Una cifra tan inabordable en cualquier estudio como inútil, porque existe 
un grupo de editoriales que son las que realmente han copado el mercado y el resto, han tenido una incidencia muy limitada. Por otro lado, sólo en el año 2016, último año del que tenemos datos hasta el momento, se publicaron en España 18.145 títulos correspondientes a la materia "Texto no universitario", que comprende los libros de Educación Infantil, Educación Primaria, Educación Secundaria Obligatoria (ESO), Bachillerato, Formación Profesional (FP) y Libros y materiales complementarios. Federación de Gremios de editores de España, Comercio interior del libro 2016 (FEDERACIÓN..., 2017).

Por esta razón, lo aconsejable, es que seleccionemos unas materias o disciplinas académicas (en función de que sus objetivos y contenidos curriculares estén relacionados con el fin de la investigación), unos niveles educativos y aquellas editoriales de mayor incidencia y difusión entre el alumnado. La razón fundamental por la que vemos necesario la elaboración de un mapa editorial es porque se tiene en cuenta la relevancia de los libros de texto en función de su difusión entre los centros educativos.

Pero no siempre se ha tenido en cuenta el criterio de la difusión o, en términos comerciales, las ventas de unos productos llamados libros de texto, con lo que la repercusión de este tipo de investigaciones entre el alumnado y el profesorado puede ser prácticamente nula. El objetivo de este estudio, lo reiteramos, es reflexionar sobre las fuentes españolas relacionadas con los libros de texto en las que los investigadores suelen basarse a la hora de mostrar la repercusión de sus estudios. Examinaremos las fuentes provenientes tanto de los organismos y entidades públicas, como las generadas por el propio sector editorial: ISBN, Estadística de la producción editorial de libros editado por el Instituto Nacional de Estadística, Registro Mercantil, los informes anuales que se editan en el Comercio exterior del libro, la plataforma Distribuidor de información del libro español en venta (Dilve), informes y estudios de investigadores e instituciones, Asociación Nacional de Editores de Libros y Material de Enseñanza (Anele), la prensa diaria y la publicación anual Comercio interior del libro en España. Este estudio puede ser muy útil para quienes 
investiguen sobre cualquier campo relacionado con los libros de texto.

La Federation of European Publishers (FEP) que agrupa a 29 miembros representantes nacionales, publica declaraciones, resoluciones, propuestas, eventos, informes, estadísticas, etc. relacionados con el sector editorial europeo de manera global (FEP, 2018). Desde esta dirección web se puede acceder a la dirección de cada uno de los miembros de esta Federación y conocer diversos informes y datos generales, anuales y nacionales. El trabajo que presentamos es posible hacerlo en cada país europeo con algunas modificaciones, en función de los datos publicados por cada federación nacional de editores.

Dentro de esta Federation of European Publishers existe un subgrupo de editores educativos que mantienen reuniones, comparten ideas, problemas y propuestas. El objetivo de este grupo subgrupo es apoyar el crecimiento de las publicaciones educativas y difundir su impacto positivo en el aprendizaje y en la enseñanza en todo el mundo. Para ello, mantiene reuniones como la celebrada en Tallin, Estonia, el pasado 5 de diciembre de 2017 con el objeto de compartir innovaciones, proponer políticas públicas sobre el rol de los editores y de los recursos editoriales en los procesos educativos, fomentar investigaciones sobre desarrollos tecnológicos que puedan impactar en las publicaciones educativas, fomentar el trabajo colaborativo entre editores y docentes, desarrollo de tecnologías digitales, etc. Aunque el enfoque de esta organización está centrado en los mercados europeos, los mensajes claves también abordan cuestiones más amplias en torno a la educación para los países en desarrollo.

\section{EJERCIENDO FUNCIONES DE ZAHORÍ}

La heurística utiliza estrategias que facilitan la reflexión y la interpretación. Los historiadores, como los zahoríes, son personas que pretenden desvelar lo que está oculto; escudriñan allí donde otros no identifican las fuentes que verdaderamente aportan un caudal significativo para aquello que se 
investiga. Es una tarea ardua, de gran sensibilidad y no siempre exitosa.

Con el boom de las editoriales de libros de texto producido en torno a Ley 14/1970 (AGENCIA ESTATAL, 1970), se produjo una transformación en el sector editorial cuyas causas ya han sido analizadas (BEAS MIRANDA; MONTES, 1998). Por una parte, se puso punto y final a unos modelos de enseñanza obsoletos que utilizaban como libros de texto para la Enseñanza Primaria las enciclopedias.

\begin{abstract}
Antonio Álvarez, maestro de Zamora, fue el autor de más éxito y dio nombre a la más conocida de estas obras, editada por la Editorial Miñón de Valladolid. De la Enciclopedia Álvarez, "intuitiva, sintética y práctica" como decía en su portada, se llegaron a vender más de treinta millones de ejemplares y llegó a copar el 80\% del mercado de enciclopedias y libros para ese nivel de la enseñanza (SANTOS ARRABAL, 2013, p. 13).
\end{abstract}

Por otra, se desarrollaron y consolidaron dos "muy grandes" editoriales así las denomina el gremio de editores a las que facturan anualmente más de sesenta millones de euros - que se han mantenido como tales desde entonces: Anaya y Santillana.

Pero ċen qué datos nos basamos para afirmar que una editorial tiene mayor o menor difusión? El sector editorial es muy opaco, fundamentalmente porque las editoriales se resisten a informar sobre la expansión de sus productos. Ninguna editorial hace públicas las cifras de libros de texto de un nivel y de una materia que ha vendido en un año concreto. Los editores, por ley, están obligados a hacer públicos algunos datos, pero no todos aquellos de los que podemos extraer informaciones globales; lo mismo sucede con otras cifras que publican organismos y entidades públicas.

La primera de las fuentes que analizamos ha sido controlada desde 1972 por el Ministerio de Educación Cultura y Deporte a través del ISBN, siglas que corresponden a las iniciales de International Standard Book Number Número Estándar Internacional de Libros o Número Internacional Normalizado 
del Libro (AGENCIA ESTATAL, 2010). Desde el 23 de diciembre de 2010, la Gestión de la Agencia Española del ISBN ha pasado a ser asumida por la Federación de Gremios de Editores de España, mientras que la titularidad de la Agencia sigue siendo ejercida por el Ministerio de Educación, Cultura y Deporte (2010). Dicho Ministerio publica anualmente desde 1988 los datos relacionados con el ISBN en la Panorámica de la edición española de libros (la publicación del año 2016 es la última editada hasta la finalización de este artículo).

Esta publicación recoge los ISBN globales, por materias y, además, en los anexos estadísticos, en la Materia o8 (Enseñanza, Educación) informa sobre las 25 mayores empresas editoras. Esta fuente no es válida para realizar un mapa editorial de los libros de texto que se utilizan en los centros educativos porque, entre otras razones y como hemos dicho, es una información global de la editorial aludiendo a libros de "Enseñanza y educación", no solo a los libros de texto. En la Panorámica de la edición española de libros (2010), por ejemplo, la Editorial MAD ocupa el puesto número uno con 2.750 ISBN. Esta editorial se dedica, fundamentalmente, a la edición de libros e eBooks para preparar oposiciones y empleo público en España. Sin embargo, la Editorial Santillana, que todos sabemos que actualmente es una de las tres grandes de España en libros de texto, no aparecía en el ranking de ese año entre las 25 primeras. En la edición de libros (2012), la editorial MAD ocupa el puesto número 16 con 253 ISBN y la Editorial Santillana el puesto número 12 con 285 ISBN. En la edición de libros (2013), la editorial MAD ocupa el puesto número cuatro con 575 ISBN, mientras que la Editorial Santillana no aparece entre las 25 editoriales seleccionadas. Con respecto a la edición de libros (2014), observamos que la editorial MAD ocupa el puesto número 10 con 349 ISBN y la Editorial Santillana está en el puesto número dos con 1.433 ISBN, es decir, es la primera vez que esta editorial está en puestos superiores a la editorial MAD. Consecuentemente, el conocimiento del número de ISBN no está relacionado con la comercialización de los libros de texto de la Enseñanza primaria o secundaria.

En el año 2016 se solicitaron 13.473 libros españoles para la asignación 
del ISBN en la materia "Enseñanza. Educación". La submateria denominada "Enseñanza secundaria. Bachillerato. Textos" ocupó el primer lugar con 5.173, seguida de "Enseñanza primaria. Textos" con 3.383; ocupa el tercer lugar la submateria "Enseñanza profesional. Textos. Temarios de oposiciones" con 1.735; en cuarto lugar, "Educación: obras generales. Métodos y Filosofía. Psicopedagogía" con 1.293 y en quinto lugar, a gran distancia, "Educación infantil. Textos" con 593 ISBN. En esta clasificación también se incluyen submaterias como "Organización de la educación y de la enseñanza. Centros", "Formación de la inteligencia y personalidad. Educación sexual”, "Enseñanza superior. Universidades y escuelas especiales", "Enseñanza de deficientes, minusválidos, inadaptados” y “Tiempo libre. Ocio" (PANORÁMICA..., 2016).

Esta fuente es muy útil para analizar de forma global datos básicos relacionados con los libros de texto como el aumento o el descenso de su producción por niveles educativos, la proporción de libros traducidos, las lenguas de publicación, las comunidades autónomas en las que más se edita, el precio medio, la cifra media de páginas por libro, la edición en distintos soportes, etc.

La segunda fuente es la que publica el Instituto Nacional de Estadística (INE), Estadística de la producción editorial de libros, y que se realizó por primera vez en 1965. Agrupa los libros según la clasificación de la Unesco. El tema número 8 (que corresponde a Educación, enseñanza, formación y distracciones) incluye además libros y folletos. Los libros se distinguen de los folletos por el número de páginas, que no ha de ser inferior a 49 en el caso de los libros, y de 5 hasta 48 páginas para los folletos (INE, 2014). Los datos que publica son muy globales y aluden al número de títulos editados en España y por comunidades autonómicas, ejemplares impresos, tirada media, etc. siendo muy útiles para obtener una visión global de la producción editorial, pero no para conocer cifras concretas que podamos vincular con determinadas editoriales y libros de texto.

La tercera fuente la podemos localizar en el Registro Mercantil, lugar donde se recogen las cifras del colectivo editor relacionadas con las cuentas cerradas de los ejercicios económicos anuales. Se han realizado algunos estudios 
basados en las cifras de negocio que las editoriales presentan en el Registro Mercantil y los publicó en la desaparecida revista Delibros en el número de septiembre o de octubre de cada año (2002, 2003, 2013). Como es lógico, las editoriales aportan cifras globales de las que se pueden analizar aspectos financieros a través de los números de sus balances; ratios de financiación, endeudamiento y liquidez; los resultados económicos y sus rendimientos, la capitalización, etc. Con esos datos, podemos observar la importancia económica de una editorial de manera global, el dominio en el mercado de los holding y de los grupos de empresas, elaborar un ranking según las cifras de venta, conocer su salud económica o establecer una comparativa entre las editoriales por su tamaño y por su volumen de ventas. En este caso, los resultados serían similares a los que aporta anualmente el Comercio Interior del Libro, pero menos precisos.

La cuarta fuente la hallamos en el Comercio exterior del libro, publicación anual que desde 1992 edita la Federación Española de Cámaras del Libro con el patrocinio del Ministerio de Educación, Cultura y Deporte. En ella, se pueden analizar las cifras de exportación y de importación por materias y, en concreto, de los libros de "Enseñanza no Universitaria". Podemos conocer de manera global, el número de ejemplares y los países receptores o emisores de mayor relevancia en esta materia. Entre los países a los que se exporta más en "Enseñanza no Universitaria” destacan México, República Dominicana, y Perú cuyas ventas en el año 2016 suponen el 38\% del total que fueron 21.603.424€, seguido de los países Estados Unidos, Chile, Portugal, Argentina y Colombia cuyas exportaciones superaron el millón de euros cada uno lo que representa el $26 \%$ del total. Es decir, la exportación de los libros de enseñanza no universitaria de los ocho países mencionados representó el 64\% del total (ASOCIACIÓN DE LAS CÁMARAS..., 2017).

En cuanto a la importación, la materia libros de "Enseñanza no Universitaria” con $487.945 €$ (lo que supone el 1,29\% del total del precio de libros importados) es la penúltima en importancia; tan sólo importa menos la materia "Diccionarios y enciclopedias" (FEDERACIÓN ESPAÑOLA DE CÁMARAS..., 
2015). Si comparamos las cifras de exportación y de importación con las de facturación en el mercado español de ese mismo año, 2016 y en el mismo sector "Texto no universitario" que fueron 856.040.00o€, observamos una desproporción desmesurada.

España exporta mucho más de lo que importa en libros de "Enseñanza no Universitaria" pero es obvio que ni exporta ni importa curricula educativos. Lo que no dicen las cifras es que aproximadamente el 90\% de lo que se importa son libros de enseñanza de Inglés de las editoriales Oxford, Cambridge, Heinemann Macmillan, Penguin, Burlington Books, etc. Y se exportan libros de texto de Español como lengua extranjera y libros de texto a los centros españoles en el extranjero. Otra de las vías de exportación son las licitaciones que obtienen los editores por haber ganado algún concurso público de alguno de los países americanos de habla española. Las grandes editoriales españolas tienen filiales, se asocian o compran editoriales en los diferentes países, pero lógicamente se editan libros de texto siguiendo los criterios curriculares impuestos por los países receptores.

En quinto lugar, otras fuentes provienen de estudios, informes, etc. de origen público, a través de las administraciones, o privado, por iniciativa fomentada por algún proyecto o alguna investigación. La Consejería de Educación de la Junta de Andalucía (2017), por ejemplo, permite conocer los libros de texto que cada centro andaluz elige cada año.

Estudios muy rigurosos han analizado el sector del libro escolar, pero de manera global. En el panorama español, por citar algún trabajo reciente, encontramos el realizado por Antonio Viñao Frago (2015), por el Centro de Investigación Manes (2017) o por Agustín Escolano Benito (1998, 2002 y 2006). A nivel internacional, el estudio de Vahl y Peres (2016). Más de 1.200 libreros pertenecientes a la Confederación Española de Gremios y Asociaciones de Libreros (Cegal), notifican los 50 libros más vendidos, pero son solo libros de lectura, no incluyen los de texto que en el mes de septiembre acaparan las ventas. Distribuidor de información del libro español en venta (Dilve) es una plataforma 
que permite la gestión y distribución de información bibliográfica y comercial del libro de forma centralizada y normalizada. Esta fuente informa de las antiguas fichas hoy llamadas metadatos: autor, título, editorial, tamaño, colección, etc. y precio, excepto el de los libros de texto que como se sabe no es fijo para los de la educación obligatoria (DILVE, 2017).

También se han publicado informes de gran repercusión sociocultural en los que no se incluía ningún estudio sobre el mapa editorial, como fue el caso del Informe que la Real Academia de la Historia (2000) publicó en ese año sobre el contenido de los libros de texto de Historia. Otros, como el publicado un año después por la Fundació Jaume Bofill (2001), parten de la premisa de que el libro de texto es un recurso al servicio del profesor y del alumno dentro del proceso de enseñanza-aprendizaje y sí se preocuparon de seleccionar las editoriales de mayor difusión en la ESO y en Bachillerato, es decir, los libros más significativos. Para lo cual, utilizaron varias fuentes de información: realizando muestreos que reflejaban las cuotas de mercado de las distintas editoriales en las diferentes comunidades autonómicas en las que centraron su análisis (Andalucía, Canarias, Cataluña, Galicia, País Vasco, Valencia, comunidades del antiguo territorio del Ministerio de Educación y Ciencia y Madrid); sondearon también diversas fuentes informativas como distribuidores, comerciales y personal de las editoriales, que, cuando informan, lo hacen de manera confidencial, etc. De manera similar podemos hablar de la tesis doctoral realizada por la profesora Erika González en la que se incluye un mapa editorial (GONZÁLEZ, 2011). Estudios como estos, en los que la validez y la fiabilidad están garantizados, son los deseados y más pertinentes, pero difíciles de realizar.

La sexta fuente proviene de los datos y documentación que aporta la Asociación Nacional de Editores de Libros y Material de Enseñanza (Anele). Esta Asociación se creó en marzo de 1978,

Desde su fundación, tiene un carácter nacional y agrupa al 96\% de los editores que publican en España libros y materiales destinados a la enseñanza reglada no universitaria, en cualquiera de las lenguas 
reconocidas por la Constitución como vehiculares de la educación. El principal objetivo de ANELE es el de representar a los editores de libros educativos ante las correspondiente entidades administrativas, así como asesorar y coordinar todas las actividades colectivas del sector (ANELE, 2018).

A través de su página web podemos extraer datos relacionados con los catálogos de libros actuales tanto en formato papel como en digital de los 24 sellos editoriales asociados en 2017, conexión con las páginas web de estas editoriales, diversos informes, sobre todo los relacionados con la evolución de los precios de los libros de texto, informes sobre el sistema de gratuidad, convenios y acuerdos de colaboración con diferentes administraciones y gobiernos autonómicos, hace públicas notas de prensa en las que se posiciona respecto a problemas que afectan al sector editorial, etc.

Las empresas que pertenecen a Anele en 2017, según su página web, son: Algaida, Anaya, Barcanova, Bromera, Bruño, Cambridge, Casals, Cruilla, Difusión, Donostiarra, Edebé, Editex, Grup Promotor, Ikaselkar, Macmillan Iberia, McGraw-Hill Interam, Oxford Univ. Press, Pearson Educación, PPC, Santillana, SM, Teide, Tekman Books y Xerais. Observamos que faltan empresas como Edelvives, Vicens Vives, Algaida, Aljibe, Erein, Everest, Laberinto, La Galera, Océano Editorial, Serbal y SGEL que otros años si han pertenecido a esta Asociación. Estas empresas reciben información de las reformas curriculares, establecen decisiones comunes como la aprobación del Código de conducta del sector editorial de libros de texto y material de enseñanza (ANELE, 2014), acuerdan una disciplina de mercado, estaban al día con las negociaciones de los precios (cuando estaban intervenidos), acuerdan las alegaciones a las distintas normas educativas, se realiza una labor de mediación entre las administraciones y el sector editorial y entre éste y los proveedores, etc.

Existen otras empresas de tamaño pequeño que no pertenecen a Anele y que no son relevantes para la confección de un mapa editorial como Arcobaleno 2.00o, Gulington Books, Difusión, Cultura Clásica, Ediciones la Ñ, Editécnicas, Editorial Donostiarra, Editorial Elzevir, Editorial Marfil, Editorial Merial, 
Editorial Pidotribo, Express Publishing, Proyecto Sur de Ediciones, etc.

Anele (2017) tiene un papel muy activo con las distintas administraciones ejerciendo de puente entre éstas y las editoriales. Cada año, en primavera, suele publicar en su página web dos catálogos (uno para los libros impresos en papel y otro para los libros digitales) que incluyen los libros disponibles para el próximo curso escolar, diferenciando título, autor, años de publicación, lengua, tipo de material, editorial, materia y tipo de educación. Las editoriales asociales han editado para el curso 2017-18, en distintas lenguas, en formato papel y para todos los niveles educativos no universitarios un total de 33.151 títulos y 13.818 en soporte digital (ANELE, 2017). La evolución de la edición digital ha sido exponencial puesto que, en 2010, primer año del que se tienen registros fiables, se editaron tan solo 110 títulos. Sin embargo, el incremento tan enorme del número de títulos en catálogo en formato digital sólo representó el 3,06\% de la facturación en papel para el curso 2016-2017 lo que significó 26.203.000€ (ANELE, 2017). Se deduce claramente que aunque existe una oferta editorial, no hay demanda. Lo importante no es el formato del libro, en papel o en digital, sino tener la posibilidad de acceder a través de web a multitud de servicios y de plataformas digitales que ofrezcan materiales alternativos y/o complementarios al libro de texto y que el profesorado, que en definitiva es quien elige los recursos educativos, opte por la opción que considere más idónea para el modelo educativo del centro.

La séptima fuente emana de las publicaciones relacionadas con los libros de texto y que a diario aparecen en la prensa de los distintos países.

Los diarios publican con mucha frecuencia noticias relacionadas con los libros de texto. La segunda quincena de agosto y en el inicio de cada curso escolar, por ejemplo, es una época propicia para que los periódicos se hagan eco del precio del material escolar, el debate sobre la gratuidad de los libros, ofertas, descuentos y promociones relacionadas con su venta, lo que las familias se ven obligadas a gastar por cada hijo en edad escolar, las iniciativas de ONG o de asociaciones que pretenden dar una segunda vida a los materiales escolares, el intercambio y 
donación de libros usados, el mercado de segunda mano, campaña de recogida de libros, becas para este fin, inversiones de las comunidades autonómicas o de los ayuntamientos para manuales escolares, etc.

Raro es el día en el que no aparecen noticias que de alguna manera están relacionada con los libros de texto como la existencia de propuestas didácticas y recursos educativos alternativos, el peso de las mochilas y su repercusión en la salud de los escolares, el comercio editorial, métodos pedagógicos y experiencias innovadoras que no utilizan libros de texto, su relación con los recursos digitales, algunas reflexiones sobre ciertos contenidos de libros de texto (en este sentido, se les suelen adjudicar de manera generalizada los males que se le achacan al sistema educativo: adoctrinamiento, manipulaciones, errores, sexismo, su carácter obsoleto, necesidad de controlarlos mediante una supervisión previa, denuncia de su carga ideológica, etc.). Todas estas cuestiones son de gran calado y muestran la relevancia del recurso educativo más utilizado en los centros educativos de España. Para Bayona (2009),

\footnotetext{
El libro de texto es la principal herramienta de los docentes y el $81,3 \%$ de ellos reconocen emplearlo bastante o mucho en la labor diaria... También los padres consideran imprescindible el libro de texto en la educación de sus hijos, tanto en los centros educativos como en el hogar (71,9\%). A su juicio, ocupa el primer lugar entre los recursos didácticos más importantes que tienen en el hogar, a mucha distancia del segundo, que es Internet (p. 25).
}

Pero estos temas no están relacionados con el objeto de nuestro estudio: fuentes para la construcción de un mapa editorial español de libros de texto.

De alguna forma, los libros de texto están presentes en los debates educativos y, casi siempre, son criticados y considerados como uno de sus males. Resulta insólito que aparezca alguna noticia que pondere los libros de texto o los valore positivamente. Existe un imaginario colectivo que los ha sacralizado durante generaciones y por eso les extraña a algunos que exista una pluralidad de editoriales y de enfoques hermenéuticos. Todavía nos cuesta aceptarlos como 
recursos educativos en manos de la comunidad educativa y de ninguna manera como algo que hay que reproducir y asumir en el proceso enseñanza aprendizaje. Por tanto, no debería ser noticia que alguien muestre su discrepancia sobre alguno de sus aspectos. Son recursos educativos y como tales, perfectamente prescindibles o utilizables de diferentes formas.

Por último, la fuente más útil porque genera un mayor caudal de información relacionado con el libro de texto y nos facilita la realización de un mapa editorial, aunque de manera global, es la que proviene de la Federación de Gremios de Editores de España quien publica anualmente desde 1989 el Comercio interior del libro en España.

En 2017 se ha publicado la 28 edición, que contiene datos correspondientes al año 2016. No es una fuente que podamos utilizar para los años anteriores a 1988, pero sí para un período bastante extenso y significativo. Durante casi treinta años, esta publicación ha experimentado cambios notables que han repercutido en una mayor información y transparencia. Así, por ejemplo, durante los primeros años, se publicaban unos datos globales con la denominación "Texto no universitario"; posteriormente, esta materia se especificó diferenciando las cifras de Educación infantil, Educación primaria, ESO, Bachillerato, FP y Libros y materiales complementarios; este último apartado incluye diccionarios, guías del profesor, Biblias, Nuevo Testamento, Evangelios, libros de comentarios de texto, libros de lectura que entran en el circuito escolar, cuadernos de vacaciones, materiales auxiliares, cuadernos de ortografía, de cálculo, atlas, etc.

En el estudio número once del Comercio interior del libro en España 1.999 se produjeron novedades importantes que fomentaban la transparencia del texto no universitario. Los datos de 1998 desglosaban las cifras especificándose en cinco apartados: Educación Infantil, Educación Primaria, ESO, FP y finalmente BUP y COU. Además, estos apartados los relacionaban con el tamaño de las empresas divididas según su facturación. Otra novedad importante supuso la inclusión de una tabla con la "Cifra de facturación según materias, por 
tamaño de empresas" (FEDERACIÓN..., 2000, p. 92). El año 2000 es el último en el que se incluyen las cifras de los libros de COU que aparecían unidas al Bachillerato y lógicamente ya aparecen las cifras en euros. Finalmente, en el estudio correspondiente al año 2005 se publican por primera vez los Ejemplares vendidos según materias, por tamaño de empresas. Esta información la consideramos muy importante para la realización de los mapas editoriales aunque se realicen de manera muy genérica, porque aunque no nos proporciona un conocimiento preciso sobre la cuota de mercado que tiene cada editorial en los diferentes niveles educativos, sí nos informa globalmente sobre el reparto de los grupos editoriales según su tamaño (empresas de tamaño muy grande, grande, medianas y pequeñas) y los niveles educativos.

Los objetivos iniciales del Comercio interior del libro en España (FEDERACIÓN..., 1996, p. 9) se podrían resumir en:

a) Que fuera informativo de la situación del sector;

b) Que fuera periódico, lo que le da una perspectiva histórica y facilita el análisis de tendencias y del resultado de políticas sectoriales;

c) Que fuera representativo lo que se ha conseguido porque prácticamente responden todas las empresas importantes;

d) Que fuera confidencial, de lo que no hay duda puesto que no aparecen los datos de identificación de las empresas.

Los datos del informe de 2017 representan a todas las empresas del universo y se obtienen a partir de la extrapolación de los datos muestrales según el gremio al que pertenecen las empresas, las materias que editan y el tramo de facturación. La ficha técnica de la investigación demuestra que estos datos son totalmente fiables y válidos y representan prácticamente a la totalidad del sector con una cantidad cercana al 90\% de la cifra de negocio de la edición de libros. En síntesis, son estudios rigurosos y que cumplen todos los requisitos de validez, fiabilidad y representatividad.

La clasificación de las empresas según su tamaño ha variado, 
fundamentalmente, debido al cambio de pesetas a euro y a la inflación. Desde el inicio de los estudios (1988) hasta el año 2000 inclusive, se establecieron cinco grupos de clasificación de las empresas según su volumen de facturación anual (Tabla 1).

Tabla 1 - Clasificación de las empresas según volumen de facturación anual.

\begin{tabular}{l|l}
\hline \multicolumn{2}{c}{ Empresas y volumen de facturación anual } \\
\hline \multicolumn{1}{c}{ Clasificación } & \multicolumn{1}{c}{ Tramo de facturación } \\
\hline Grande & Más de 3.00o millones de ptas. \\
\hline Media grande & De 1.000 a 3.00o millones de ptas. \\
\hline Media media & De 400 a 999 millones de ptas. \\
\hline Media pequeña & De 100 a 399 millones de ptas. \\
\hline Pequeña & Menos de 100 millones de ptas. \\
\hline
\end{tabular}

Fuente: Federación de Gremios de Editores de España (1999, p. 13).

En el estudio del año 2001 se hizo la misma clasificación, pero convertida en euros y desde 2002 se hizo una nueva, que es la que se mantiene en la actualidad (Tabla 2).

Tabla 2 - Clasificación de las empresas según volumen de facturación anual.

\begin{tabular}{l|l}
\hline \multicolumn{2}{c}{ Empresas y volumen de facturación anual } \\
\hline \multicolumn{1}{c}{ Clasificación } & \multicolumn{1}{c}{ Tramo de facturación } \\
\hline Muy grande & Más de 60.000.000 $€$ \\
\hline Grande & De 18.000.000 a 60.000.000 $€$ \\
\hline Mediana & De 2.400.000 a 18.000.000 € \\
\hline Pequeña & Menos de 2.400.000 $€$ \\
\hline
\end{tabular}

Fuente: Federación de Gremios de Editores de España (2002, p. 17). 
La estructura empresarial de todo el sector editorial, no solo el de los libros de texto, demuestra cada año:

- que existe un número reducido de muy grandes empresas (7 en el año 2016) que representan el 39,3\% de la facturación total;

- 16 editoriales grandes con un 22,3\% de facturación total;

- 108 medianas con un 25,9\% de facturación;

- y un gran número de editoriales pequeñas, 596, que, aunque enriquecen de manera muy significativa el panorama cultural de la edición, sin embargo, son las más inestables porque generan un mayor número de altas y de bajas. Su facturación sólo alcanza el 12,5 del total de 2.317,20 millones de euros, que fue la cifra de facturación de todo el sector editorial en el mercado interior (FEDERACIÓN..., 2017). Aunque las cifras que hemos mencionado son las de la última edición del Comercio interior del libro en España, las deducciones de los años anteriores son muy similares.

Una de las observaciones que consideramos más significativas es que a pesar de que la suma de las empresas de tamaño muy grande y grande representan solo el 1,44\% del conjunto de empresas, editaron en el año 2016 un total de 29.117 títulos (lo que equivale al 35,8\%) y un 59,7\% de la facturación de todo el sector (FEDERACIÓN..., 2017). La rentabilidad y salida comercial de las empresas muy grandes y grandes se manifiesta también analizando las cifras de facturación de ambos grupos en el mercado interior, que alcanzaron los 1.427,38 millones de euros en 2016, lo que equivale al 61,6\% de las cifras de todo el sector editorial. Teniendo en cuenta los datos anteriores es evidente que existe un reducido grupo de empresas que dominan el sector editorial.

A pesar de ser un concepto muy relativo y poco significativo para nuestro 
objetivo, también se puede analizar la tirada media de ejemplares por grupo de editoriales en los niveles de Infantil, Primaria, ESO, Bachillerato y FP. Estas cifras se obtienen dividiendo el número total de ejemplares editados, entre el número total de títulos. La tirada media de los años 1999 (primer año del que se publica este dato y que se inserta en el informe del año 2002), 2005, 2009, 2014 y 2016 del total de libros de texto no universitarios es, respectivamente, 5.046/4.214/4.269/2.832 y 2788 ejemplares (FEDERACIÓN..., 2002, 2006, 2010, 2015, 2017). Lógicamente, las tiradas medias de las grandes empresas son superiores a las del resto y las de Infantil, Primaria y Secundaria a las de Bachillerato y Formación Profesional.

Nos sorprende el número de títulos editados cada año; en 2016, en el apartado de texto no universitario, se editaron 18.145 títulos, ocupando el primer lugar los títulos de Educación primaria, 7.033 (FEDERACIÓN..., 2017). En ese mismo año y en el apartado Texto no universitario, existían 88.637 títulos vivos en catálogo, es decir, que se podían comercializar. En este mismo apartado, también destacamos la enorme diferencia entre el número de ejemplares producidos (50.586.00o ejemplares) y los vendidos (44.143.00o ejemplares) (FEDERACIÓN..., 2015), lo que da idea del volumen de libros que quedan en estocaje o bien se desechan y se destruyen.

El incremento de títulos editados cada año y los vivos en catálogo están relacionados, fundamentalmente, con las novedades curriculares porque los ciclos de producción son mayores cuando se implanta una reforma educativa o un cambio de normativa curricular. Cuando no se dan estos cambios, baja la edición. Por otro lado, cuando una empresa considera que puede crecer, produce nuevos productos. Además, cuando hay inflación, los estocajes se revalorizan y no se tiran. Más o menos el 30\% de la producción de las empresas editoras se queda vivo en catálogo, como excedente de distintos libros que no caducan. Los fracasos editoriales sí se desechan.

Las causas del incremento o bajada de los títulos editados dependen de: 
- Las diversas políticas y frecuentes reformas educativas que fomentan una inestabilidad curricular;

- La descentralización educativa y el Estado de las autonomías que provocan un curriculum diverso y plural. Si al principio de la década de los ochenta se editaban unos 1.800 títulos anuales, en el año 2016 se editaron 18.145 y desde 2003 se vienen sobrepasando los 16.000 títulos en el sector de libro de texto no universitario de manera continua (FEDERACIÓN..., 2017). La proliferación de una normativa educativa autonómica ha generado una gran diversidad en la concreción curricular que repercute en el sector editorial;

- La realidad plurilingüística española;

- El aumento o disminución de la población escolar;

- El aumento de la enseñanza en centros bilingües;

- La multitud de títulos indica una riqueza de oferta editorial generada con el fin de captar una cuota de mercado cada vez más impenetrable para las pequeñas empresas y más competitiva para las medianas y las grandes;

- En general, el mayor número de títulos vivos en catálogo depende más de las editoriales medianas y pequeñas que de las grandes y muy grandes. Sin embargo, analizando la edición de títulos anuales del sector libros de texto no universitario, el dominio de las empresas muy grandes y grandes (11.833 títulos) es abrumador respecto a las medianas y pequeñas (6.362 títulos) (FEDERACIÓN..., 2017). Esto quiere decir que las editoriales de mayor tamaño liquidan más fácilmente sus estocajes;

- Otras razones que afectan a la edición son la inseguridad del mercado, la financiación autonómica, el sistema de ayudas para la compra de los libros de texto, la estacionalidad tan reducida (prácticamente finales 
de agosto y principios de septiembre) de las ventas de libros, etc.;

- Con el acuerdo del Código de Conducta (ANELE, 2014), que entró en vigor el 2 de abril de 2014, esta competitividad empresarial se basa más en criterios curriculares, es decir, en la calidad del producto, que en los obsequios de las editoriales a los centros y al profesorado, que facilitaban la compra de voluntades y de decisiones educativas.

Pero donde se percibe la brecha de la incidencia de las empresas según su tamaño es analizando las cifras de facturación y el número de ejemplares vendidos.

Los datos que nos parecen más significativos para la realización de un mapa editorial de cuantos se publican en el Comercio interior del libro de los últimos nueve años (antes no se incluían en estos estudios) son los relacionados con el número de "Ejemplares vendidos según materias por tamaño de empresas" y la "Cifra de facturación según materias por tamaño de empresas". Nosotros hemos recogido los datos del primer y último año que se han publicado hasta el momento; 2008 por ser un año intermedio que supone el fin del crecimiento del sector editorial antes de la crisis económica actual y 2011 para observar ciclos de tres años (Tabla 3).

Tabla 3 - Porcentajes anuales del número de ejemplares vendidos según niveles educativos, por tamaño de empresas.

\begin{tabular}{l|l|l|l|l|l|l|l|l|l|l}
\hline \multicolumn{6}{c}{ Ejemplares vendidos según niveles educativos, por tamaño de empresas } \\
\hline & \multicolumn{3}{c}{ Muy grandes } & \multicolumn{5}{c}{ Grandes } \\
\cline { 2 - 12 } & $\mathbf{2 0 0 5}$ & $\mathbf{2 0 0 8}$ & $\mathbf{2 0 1 1}$ & $\mathbf{2 0 1 4}$ & $\mathbf{2 0 1 6}$ & $\mathbf{2 0 0 5}$ & $\mathbf{2 0 0 8}$ & $\mathbf{2 0 1 1}$ & $\mathbf{2 0 1 4}$ & $\mathbf{2 0 1 6}$ \\
\hline Primaria & 56,5 & 54,1 & 67,3 & 57,69 & 61,28 & 20,5 & 35,4 & 19,5 & 21,42 & 19,8 \\
\hline ESO & 44 & 41,4 & 51,9 & 33,55 & 44,28 & 26 & 43,8 & 27,8 & 32,05 & 19,8 \\
\hline Bachillerato & 29 & 45,4 & 41,9 & 25,79 & 45,36 & 32,2 & 38,3 & 33,6 & 25,88 & 12,86 \\
\hline $\begin{array}{l}\text { Total LT no } \\
\text { universitario }\end{array}$ & 44,5 & 43,4 & 55,15 & 45,70 & 52,5 & 26 & 40,2 & 24,6 & 22,88 & 19,5 \\
\hline
\end{tabular}




\begin{tabular}{l|l|l|l|l|l|l|l|l|l|l}
\hline \multicolumn{7}{c}{ Ejemplares vendidos según niveles educativos, por tamaño de empresas } \\
\hline & \multicolumn{7}{c}{ Medianas } & \multicolumn{5}{c}{ Pequeñas } \\
\cline { 2 - 13 } & $\mathbf{2 0 0 5}$ & $\mathbf{2 0 0 8}$ & $\mathbf{2 0 1 1}$ & $\mathbf{2 0 1 4}$ & $\mathbf{2 0 1 6}$ & $\mathbf{2 0 0 5}$ & $\mathbf{2 0 0 8}$ & $\mathbf{2 0 1 1}$ & $\mathbf{2 0 1 4}$ & $\mathbf{2 0 1 6}$ \\
\hline Primaria & 17 & 8,2 & 19,9 & 20,08 & 17,6 & 6 & 2,3 & 1,3 & 1,20 & 1,39 \\
\hline ESO & 25 & 11 & 17,1 & 30,62 & 32,4 & 5 & 3,8 & 3,2 & 3,77 & 3,5 \\
\hline Bachillerato & 31,8 & 11,6 & 19,4 & 44,52 & 35,21 & 7 & 4,7 & 5,1 & 3,84 & 6,57 \\
\hline $\begin{array}{l}\text { Total LT no } \\
\text { universitario }\end{array}$ & 24 & 11,5 & 16,8 & 26,91 & 24,75 & 5,5 & 4,9 & 3,5 & 4,52 & 3,28 \\
\hline
\end{tabular}

Fuente: Elaboración propia a partir de los datos extraídos de la Federación de Gremios de Editores de España (2006, p. 129; 2009, p. 136; 2012, p. 160; 2015, p. 160; 2017, p. 160).

Observando los porcentajes del total de libros de texto no universitario de Primaria, ESO y Bachillerato que aparecen en la tabla número 3, deducimos que las editoriales de tamaño muy grande aunque acaparan las ventas no lo hacen de manera abrumadora en todos los niveles educativos. En el año 2016, las cifras correspondientes al número de ejemplares vendidos por las tres editoriales muy grandes, es decir, las que venden más de sesenta millones de euros anuales, fueron: en Primaria 12.369.000, en la ESO 3.764.000, en Bachillerato 1.658.000 $\mathrm{y}$ de los 44.143.00o de ejemplares vendidos en total de libro de texto no universitario, 23.177.00o los vendieron las SM, Santillana y Anaya.

Se puede decir que las empresas de libros de texto evolucionan de manera que las posibilidades de concentración son cada vez mayores. Sin embargo, no ha habido un incremento significativo de esta concentración desde hace 30 años manteniéndose una distribución casi estable de las cuotas de mercado. En Francia, una sola editorial, Hachette, sobrepasa el 60\%. Allí, el mercado está mucho más concentrado.

A las tres empresas muy grandes, le siguen las grandes editoriales: Oxford University Press, Edebé, Edelvives y Vicens Vives. Las medianas: Teide, Pearson, McGraw-Hill, Macmillan. Las pequeñas, el resto de las editoriales: Algaida, Casals, Ediciones del Laberinto, Ediciones del Serbal, Editex, (sección de libros de texto no universitario), Pearson, La Galera, etc. (GONZÁLEZ, 2011). 
Teniendo en cuenta las cifras publicadas por la fuente que estamos analizando, podemos afirmar de manera muy global, que las empresas editoriales del sector Texto no universitario muy grandes facturan aproximadamente cerca del 50\%, las grandes entre el $25 \mathrm{y}$ el $30 \%$, las medianas el $15 \mathrm{y}$ el $20 \%$ y las pequeñas rondan el $5 \%$ restante.

En estos momentos, con la reducción del mercado debido a las consecuencias de la crisis económica, tienden a desaparecer pequeñas editoriales y las medianas también sufren las consecuencias facturando a niveles de hace más de cinco años. Vivimos una situación económica crítica que favorece la concentración debido a la necesidad que tienen las empresas de conservar una cuota de mercado que mantenga los niveles de facturación y unos beneficios que las hagan viables económicamente. Cuando el mercado se contrae, necesitan ampliar su cuota, lo que resulta muy complicado. Las grandes empresas de libros de texto españolas lo gestionan de diferentes maneras: Anaya compra empresas, como Bruño; Santillana, invierte mejorando sus productos; SM, tiene una gran solidez porque sus beneficios los canaliza hacia su Fundación, no los transfiere a ninguna otra empresa.

Por niveles educativos, observamos claramente que la concentración de las editoriales es mayor en los niveles más bajos del sistema educativo y va disminuyendo a medida que subimos los niveles. Cuando se produce un incremento de las ventas en las muy grandes y en las grandes empresas, lógicamente repercute en la facturación y subsistencia de las medianas y pequeñas que son quienes aportan mayor pluralidad curricular (Tabla 4).

Tabla 4 - Porcentajes anuales de las cifras de facturación según niveles educativos por tamaño de empresas.

\begin{tabular}{c|c|c|c|c|c|c|c|c|c|c|}
\hline \multicolumn{3}{|c}{ Porcentajes por facturación según niveles educativos por tamaño de empresa } \\
\hline & \multicolumn{5}{c|}{ Muy grandes } & \multicolumn{5}{c}{ Grandes } \\
\cline { 2 - 11 } & $\mathbf{2 0 0 5}$ & $\mathbf{2 0 0 8}$ & $\mathbf{2 0 1 1}$ & $\mathbf{2 0 1 4}$ & $\mathbf{2 0 1 6}$ & $\mathbf{2 0 0 5}$ & $\mathbf{2 0 0 8}$ & $\mathbf{2 0 1 1}$ & $\mathbf{2 0 1 4}$ & $\mathbf{2 0 1 6}$ \\
\hline Primaria & 55,2 & 65,5 & 66,7 & 58,82 & 64,4 & 22,3 & 27 & 19 & 20,66 & 16,15 \\
\hline
\end{tabular}




\begin{tabular}{l|l|l|l|l|l|l|l|l|l|l}
\hline \multicolumn{3}{|c}{ Porcentajes por facturación según niveles educativos por tamaño de empresa } \\
\hline & \multicolumn{5}{c}{ Muy grandes } & \multicolumn{5}{c}{ Grandes } \\
\cline { 2 - 12 } & $\mathbf{2 0 0 5}$ & $\mathbf{2 0 0 8}$ & $\mathbf{2 0 1 1}$ & $\mathbf{2 0 1 4}$ & $\mathbf{2 0 1 6}$ & $\mathbf{2 0 0 5}$ & $\mathbf{2 0 0 8}$ & $\mathbf{2 0 1 1}$ & $\mathbf{2 0 1 4}$ & $\mathbf{2 0 1 6}$ \\
\hline ESO & 41,3 & 40,4 & 48 & 38,98 & 46,22 & 25,5 & 42,2 & 27 & 28,84 & 17,18 \\
\hline Bachillerato & 26,6 & 35,4 & 46 & 29,89 & 46,3 & 31,5 & 42,3 & 29,8 & 24,40 & 11,26 \\
\hline $\begin{array}{l}\text { Total LT no } \\
\text { universitario }\end{array}$ & 46,3 & 43,6 & 52,7 & 47,06 & 53,7 & 25,2 & 34,5 & 25,9 & 21,62 & 17,25 \\
\hline
\end{tabular}

\begin{tabular}{l|l|l|l|l|l|l|l|l|l|l}
\hline \multicolumn{1}{|c}{ Porcentajes por facturación según niveles educativos por tamaño de empresa } \\
\hline & \multicolumn{9}{c}{ Medianas } & \multicolumn{5}{c}{ Pequeñas } \\
\cline { 2 - 12 } & $\mathbf{2 0 0 5}$ & $\mathbf{2 0 0 8}$ & $\mathbf{2 0 1 1}$ & $\mathbf{2 0 1 4}$ & $\mathbf{2 0 1 6}$ & $\mathbf{2 0 0 5}$ & $\mathbf{2 0 0 8}$ & $\mathbf{2 0 1 1}$ & $\mathbf{2 0 1 4}$ & $\mathbf{2 0 1 6}$ \\
\hline Primaria & 15 & 8,3 & 12,6 & 19,15 & 17,98 & 7,5 & 3,2 & 1,7 & 1,37 & 1,45 \\
\hline ESO & 23,7 & 11,8 & 20,3 & 29,23 & 33,46 & 9,5 & 5,6 & 4,7 & 2,94 & 3,14 \\
\hline Bachillerato & 28,4 & 13,4 & 16,9 & 42,22 & 35,95 & 13,5 & 8,9 & 7,3 & 3,49 & 6,48 \\
\hline $\begin{array}{l}\text { Total LT no } \\
\text { universitario }\end{array}$ & 21 & 13,5 & 16 & 28,09 & 25,8 & 7,5 & 8,4 & 5,4 & 3,21 & 3,18 \\
\hline
\end{tabular}

Fuente: Elaboración propia a partir de los datos extraídos de la Federación de Gremios de Editores de España (2006, p. 128; 2009, p. 134; 2012, p. 158; 2015, p. 158; 2017, p. 158).

Lógicamente, las cifras de facturación encajan con el análisis que hemos realizado al comentar la tabla del número de ejemplares vendidos. En el año 2005 se facturaron aproximadamente 698.840.00o€ en el sector de libros de texto no universitario; en 2008, 898.280.000€, en 2011, 868.010.000€, en 2014, 748,640.0oo€ y en el año 2016, 856.040.ooo€ (FEDERACIÓN..., 2009, 2012, 2015, 2017). En dinero corriente, las cifras se han ido incrementando hasta 2008; sin embargo, a partir de ese año la crisis ha afectado de manera notable a la facturación global del sector editorial y también a los libros de texto no universitario produciéndose un descenso mayor en la facturación de la ESO, Bachillerato y de los libros y materiales complementarios. Por tamaño de empresas, las menos afectadas son proporcionalmente las muy grandes y las pequeñas y, en las grandes y medianas, el descenso es más notorio. En la ESO, por ejemplo, en 2008 las grandes editoriales facturaron 110.580.000€, en 2011, 
57.890.00o€, en 2014 descendió la cifra a 45.580.00o€ y en 2016, 34.370.00o€; en esos mismos años y en el mismo nivel educativo, las muy grandes facturaron 104.490.000€, 107.580.000€, 61.600.000€ y 92.460.000€ respectivamente (FEDERACIÓN..., 2009, 2012, 2015, 2017).

\section{CONCLUSIONES}

Los historiadores debemos analizar las fuentes adecuadas y extraer de ellas unas conclusiones razonablemente pertinentes. Desafortunadamente no ha sucedido así con estudios que han pretendido interpretar unas fuentes que reflejan datos y cifras relacionadas con los manuales escolares. El sector editorial en general, y el del libro de texto en particular, son opacos y se muestran reticentes a la hora de desvelar todos los datos que podrían orientarnos a la hora de realizar un mapa editorial. Esto no justifica que intentemos construirlo basándonos en datos que ni son útiles, ni significativos para este fin, como sucede con las cifras proporcionadas por el Ministerio de Cultura en su publicación anual Panorámica de la edición española de libros que está relacionada con los ISBN; de manera parecida, con la publicación anual del INE Estadística de la producción editorial de libros, los datos que podemos extraer del Registro Mercantil y de la publicación anual Comercio exterior del libro. Todas estas fuentes, al igual que otras que hemos citado, nos aportan datos globales de las editoriales; pero no podemos extraer conclusiones erróneas ni relacionarlas con libros de texto concretos, de una materia y de un nivel determinado. Ninguno de esos ámbitos se pueden extraer de ellas; en cambio son muy valiosas para otros objetivos como, por ejemplo, para analizar la relevancia y evolución, en su conjunto, de toda la producción de las editoriales más importantes en el plano comercial.

Los datos más significativos que hoy día podemos utilizar para la construcción de un mapa editorial son los publicados anualmente por la Federación de gremios de editores de España en el Comercio interior del libro. 
Esta publicación es relativamente reciente (desde 1988) y, como hemos dicho, desglosa las cifras por niveles educativos e incluye los ejemplares vendidos por materias y por tamaño de empresas desde 2005. Reiteramos que es una fuente totalmente fiable, válida y representativa.

Otras contribuciones para la realización de un mapa editorial fiable, es decir, que reflejen la difusión real de los manuales entre la comunidad escolar proceden de estudios realizados puntualmente como el de la Fundació Jaume Bofill (SEGURA, 2001), o el elaborado por González (2011). Estudios muy arduos de acometer, pero cuya validez y fiabilidad están probadas.

Con las reflexiones que hemos aportado consideramos que esclarecemos la utilidad de cada una de las fuentes mencionadas y advertimos de la coherencia o no entre el fin para el que se han editado y el uso que se hace de ellas. Los mapas editoriales en el sector del Texto no universitario son imprescindibles si lo que se pretende es reflexionar sobre la incidencia curricular de cualquier libro de texto. Obviamente no tiene la misma repercusión el contenido de un libro de texto de una editorial muy grande que el de una pequeña cuyas ventas sean reducidas.

Indicamos, por último, que en la actualidad los centros educativos eligen los libros en función del proyecto educativo que presentan las editoriales. Los libros de texto han experimentado una gran transformación desde la década de los setenta y una de las más recientes, junto con la incorporación de los recursos digitales a los centros, ha sido que las editoriales lo que diseñan y ofrecen al profesorado son los proyectos educativos. La programación de libros sueltos ha quedado obsoleta. Esta innovación facilita la confección de un mapa editorial al mismo tiempo que ofrece nuevas perspectivas de análisis de los libros de texto cobrando protagonismo no tanto el libro de texto individual, sino el proyecto en su conjunto en el que comparten protagonismo libros de texto, recursos digitales, otros materiales impresos y páginas web.

Finalmente y como bien sugiere Martínez: 


\begin{abstract}
Evidentemente, los trabajos realizados sobre dimensiones tales como el contenido, las editoriales, la gratuidad, el uso del género, etc., son necesarios para comprender este importante recurso, pero es en el aula, en la realidad cotidiana, cuando el libro de texto se muestra tal como es, señalando sus virtudes y carencias (2016, p. 71).
\end{abstract}

\title{
REFERENCIAS
}

AGENCIA ESTATAL. Ley 14/1970, de 4 de agosto, General de Educación y Financiamiento de la Reforma Educativa. 6 de agosto de 1970. Jefatura del Estado. Boletín Oficial del Estado. Disponible en:

https://www.boe.es/buscar/doc.php?id=BOE-A-1970-852. Consultada en: ene. 2018.

AGENCIA ESTATAL. Resolución de 15 de julio de 2010, de la Secretaría General Técnica, por la que se publica el Convenio de colaboración con la Comunidad Autónoma de Cataluña y la Federación de Gremios de Editores de España, para el desarrollo en España del Sistema International Standard Book. Ministerio de Cultura. 3 de agosto de 2010. Boletín Oficial del Estado. Disponible en: https://www.boe.es/diario_boe/txt.php?id=BOE-A-2010-12474. Consultada en: ene. 2018.

ANELE. Asociación Nacional de Editores de Libros y Material de Enseñanza (Anele). Disponible en: http://anele.org/. Consultada en: 14 ene. 2018.

ANELE. Código de conducta del sector editorial de libros de texto y material de enseñanza. Asociación Nacional de Editores de Libros y Material de Enseñanza (Anele). 2014. Disponible en: http://anele.org/wpcontent/uploads/2012/o3/CDC-ABRIL-2014-Unificado.pdf. Consultada en: 14 ene. 2018.

ANELE. Juncta Directiva. Asociación Nacional de Editores de Libros y Material de Enseñanza (Anele). 2018. Disponible en: https://anele.org/laasociacion/junta-directiva/ Consultada en: 14 ene. 2018.

ASOCIACIÓN DE LAS CÁMARAS DEL LIBRO DE ESPAÑA. Comercio exterior del libro 2016. Madrid: Asociación de las Cámaras del Libro de España, 2017. p. 39.

BAYONA, Bernardo. Reflexiones y propuestas sobre políticas de 
gratuidad de los libros de texto. Madrid: Anele. Informe privado, 2009.

BEAS MIRANDA, Miguel; MONTES, Soledad. El boom de la edición escolar. Producción, comercio y consumo de libros de enseñanza. In: ESCOLANO BENITO, Agustín (ed.). Historia ilustrada del libro escolar en España. Del Franquismo a la Restauración Democrática. Madrid: Fundación Germán Sánchez Ruipérez, 1998. p. 73-102.

CENTRO DE INVESTIGACIÓN MANES. Proyecto Manes. Disponible en: http://linhd.uned.es/p/proyecto-manes/. Consultada en: 14 ene. 2018.

CONSEJERÍA DE EDUCACIÓN, JUNTA DE ANDALUCÍA. Consulta selección de libros de texto por centro para el curso 2015/2016. Disponible en:

http://www.juntadeandalucia.es/educacion/vscripts/libros/index.asp. Consultada en: 14 ene. 2018.

DILVE. Distribuidor de información del libro español en venta (Dilve). Disponible en: https://www.dilve.es/dilve/dilveweb/index_dilve.jsp. Consultada en: 14 ene. 2018.

ESCOLANO BENITO, Agustín. Historia ilustrada de la escuela: dos siglos de perspectiva histórica. Madrid: Fundación Germán Sánchez Ruipérez, 2006.

\section{ESCOLANO BENITO, Agustín. Historia ilustrada del libro escolar en}

España: de la posguerra a la reforma educativa. Madrid: Fundación Germán Sánchez Ruipérez, 1998.

ESCOLANO BENITO, Agustín. The historical codification of the manualistics in Spain. Paedagógica Historica: International journal of the history of education, n. 38, p. 11-24, 2002.

FEDERACIÓN DE GREMIOS DE EDITORES DE ESPAÑA. Comercio interior del libro 1995. Madrid: Federación de Gremios de Editores de España, 1996.

FEDERACIÓN DE GREMIOS DE EDITORES DE ESPAÑA. Comercio interior del libro 1999. Madrid: Federación de Gremios de Editores de España, 2000.

FEDERACIÓN DE GREMIOS DE EDITORES DE ESPAÑA. Comercio interior del libro 2oo1. Madrid: Federación de Gremios de Editores de España, 2002.

FEDERACIÓN DE GREMIOS DE EDITORES DE ESPAÑA. Comercio 
interior del libro 2005. Madrid: Federación de Gremios de Editores de España, 2006.

FEDERACIÓN DE GREMIOS DE EDITORES DE ESPAÑA. Comercio interior del libro 20o8. Madrid: Federación de Gremios de Editores de España, 2009.

FEDERACIÓN DE GREMIOS DE EDITORES DE ESPAÑA. Comercio interior del libro 2009. Madrid: Federación de Gremios de Editores de España, 2010.

FEDERACIÓN DE GREMIOS DE EDITORES DE ESPAÑA. Comercio interior del libro 2o11. Madrid: Federación de Gremios de Editores de España, 2012.

FEDERACIÓN DE GREMIOS DE EDITORES DE ESPAÑA. Comercio interior del libro 2014. Madrid: Federación de Gremios de Editores de España, 2015.

FEDERACIÓN DE GREMIOS DE EDITORES DE ESPAÑA. Comercio interior del libro 2016. Madrid: Federación de Gremios de Editores de España, 2017.

FEDERACIÓN ESPAÑOLA DE CÁMARAS DEL LIBRO. Comercio exterior del libro 2014. Madrid: Asociación de las Cámaras del Libro de España, 2015.

FEP. La Federation of European Publishers (FEP). Disponible en: $<$ https://www.fep-fee.eu >. Consultada en: ene. 2018.

GONZÁLEZ, Erika. Ciudadanía, identidades complejas y cultura política en los manuales escolares andaluces de Educación para la ciudadanía y los Derechos Humanos. 2011. 472 p. Tesis doctoral (Doctorado en Pedagogía) - Universidad de Granada, Granada, España, 2011.

INE. Nota de prensa. Estadística de la producción editorial año 2014. Instituto Nacional de Estadística. 2014. Disponible en: http://www.ine.es/dyngs/INEbase/es/operacion.htm?c=Estadistica_C\&cid=12 $54736176767 \&$ menu=ultiDatos\&idp=1254735573113. Consultada en: 14 dic. 2017.

MARTÍNEZ, Nicolás. El uso del libro de texto de historia de España en bachillerato: entre el aula y la casa. Revista História da Educação, v. 20, n. 50, p. 69-93, sept./dic. 2016.

MINISTERIO DE CULTURA. Panorámica de la edición española de 
libros 2010. Madrid: Observatorio de la Lectura y el Libro, 2011. p. 163. MINISTERIO DE EDUCACIÓN, CULTURA Y DEPORTE. Panorámica de la edición española de libros 2011. 2012. Disponible en:

http://www.mcu.es/libro/docs/panoramica2011.pdf. Consultada en: 14 dic. 2017.

MINISTERIO DE EDUCACIÓN, CULTURA Y DEPORTE. Panorámica de la edición española de libros 2012. Análisis sectorial del libro. 2013.

Disponible en:

http://www.mcu.es/libro/docs/MC/Observatorio/pdf/PANORAMICA2012.pdf. Consultada en: 14 dic. 2017.

MINISTERIO DE EDUCACIÓN, CULTURA Y DEPORTE. Panorámica de la edición española de libros 2013. Análisis sectorial del libro. 2014.

Disponible en:

http://www.lamoncloa.gob.es/espana/eh15/culturaydeporte/Documents/Panor amica\%20edicion\%2olibros\%202013.pdf. Consultada en: 14 dic. 2017.

MINISTERIO DE EDUCACIÓN, CULTURA Y DEPORTE. Panorámica de la edición española de libros 2014. Análisis sectorial del libro. 2015.

Disponible en: http://www.cegal.es/wp-

content/uploads/2016/05/Panor\%C3\%A1mica-de-la-Edici\%C3\%B3nEspa\%C3\%B1ola-de-Libros-2014.pdf. Consultada en: 14 dic. 2017.

MINISTERIO DE EDUCACIÓN, CULTURA Y DEPORTE. Panorámica de la edición española de libros, 2016. Disponible en: https://www.cegal.es/wpcontent/uploads/2017/o9/Panor\%C3\%A1mica-de-la-Edici\%C3\%B3nEspa\%C3\%B1ola-de-Libros-2016.pdf. Consultada en: 14 dic. 2017.

REAL ACADEMIA DE LA HISTORIA. Informe sobre los textos y cursos de Historia en los centros de Enseñanza Media. Madrid. 23 jun. 2000. Filosofia en Español. Disponible en: http://www.filosofia.org/his/h20ooah.htm. Consultada en: 14 dic. 2017.

SANTOS ARRABAL, Maurício. El pasado hasta casi el presente de los manuales escolares. In: BEAS MIRANDA, Miguel (ed.). Ciudadanías e identidades en los manuales escolares (1970-2012). Sevilla: Díada Editora, 2013. p. 11-27.

SEGURA, Antoni. Els llibres d'història, l'ensenyament de la historia i altres històries. Barcelona: Fundació Jaume Bofill, 2001.

VAHL, Mônica Maciel; PERES, Eliane. As disputas editoriais no campo do programa do livro didático para o Ensino Fundamental do Instituto Nacional do 
Livro - Plidef/INL (1971-1976). Revista História da Educação, v. 20, n. 50, p. 219-241, sep./dic. 2016.

VIÑAO FRAGO, Antonio. El libro escolar. In: MARTÍNEZ MARTÍN, Jesús Antonio (dir.). Historia de la edición en España 1939-1975. Madrid: Marcial Pons, 2015. p. 682-699.

MIGUEL BEAS MIRANDA es profesor jubilado de la Facultad de Ciencias de la Educación de la Universidad de Granada (UGR), España. Maestro, licenciado en Historia, licenciado en Pedagogía y doctor en Filosofía y Letras - Ciencias de la Educación. Pertenece a la Sociedad Española de Historia de la Educación (Sedhe) y a la Sociedad para el Estudio del Patrimonio Histórico-Educativo (Sephe). Con más de 40 años de experiencia docente en todos los niveles educativos, sus investigaciones se relacionan con libros de texto, ciudadanía, identidades, curriculum, exclusión social.

E-mail: mbeas@ugr.es

(iD) http://orcid.org/0000-0002-9889-7658

ERIKA GONZÁLEZ GARCÍA es doctora en Pedagogía por la Universidad de Granada (UGR), España. En la actualidad desarrolla su labor docente como profesora ayudante doctora en el Departamento de Pedagogía. Sus principales líneas de investigación giran en torno al estudio de la historia de la educación: análisis de manuales escolares, ciudadanía, identidades, estudio del patrimonio educativo, identidades y emociones.

E-mail: erikag@ugr.es

(D) http://orcid.org/0000-0003-2465-7288

Recebido em: 15 de fevereiro de 2018

Aprovado em: 18 de dezembro de 2018

Revista História da Educação - RHE

Associação Sul-Rio-Grandense de Pesquisadores em História da Educação - Asphe

Artigo de acesso aberto distribuído nos termos de licença Creative Commons. 